\title{
Concealed Weapon Detection Using Color Image Fusion
}

\author{
Zhiyun Xue, Rick S. Blum \\ Electrical and Computer Engineering Department \\ Lehigh University \\ Bethlehem, PA, U.S.A. \\ rblum@eecs.lehigh.edu
}

\begin{abstract}
Image fusion is studied for detecting weapons or other objects hidden underneath a person's clothing. The focus of this paper is to develop a new algorithm to fuse a color visual image and a corresponding IR image for such a concealed weapon detection application. The fused image obtained by the proposed algorithm will maintain the high resolution of the visual image, incorporate any concealed weapons detected by the IR sensor, and keep the natural color of the visual image. The feasibility of the proposed fusion technique is demonstrated by some experimental results.
\end{abstract}

Keywords: Image fusion, concealed weapon detection, color image, IR image, sensor fusion.

\section{Introduction}

Concealed weapon detection (CWD) is an increasingly important topic in the general area of law enforcement and it appears to be a critical technology for dealing with terrorism, which appears to be the most significant law enforcement problem for the next decade. Existing image sensor technologies for CWD applications include thermal/infrared (IR), millimeter wave (MMW), and visual. Since no single sensor technology can provide acceptable performance in CWD applications, image fusion has been identified as a key technology to achieve improved CWD procedures [1-3]. Image fusion is a process of combining complementary information from multiple sensor images to generate a single image that contains a more accurate description of the scene than any of the individual images. While MMW sensors have many advantages, the availability of low cost IR technology makes the study of fusing visual and IR images of great interest. This is the focus of this paper.

In our current work, we are interested in using image fusion to help a human or computer in detecting a concealed weapon using IR and visual sensors. One example is given in Figure 1. Figure 1(a) shows the color visual image and (b) shows the corresponding IR image. The visual and IR images have been aligned by image registration. We observe that the body is brighter than the background in the IR image. Further the background is almost black and shows little detail because of the high thermal emissivity of body. The weapon is darker than the surrounding body due to a temperature difference between it and the body (it is colder than human body). The resolution in the visual image is much higher than that of the IR image, but there is no information on the concealed weapon in the visual image.

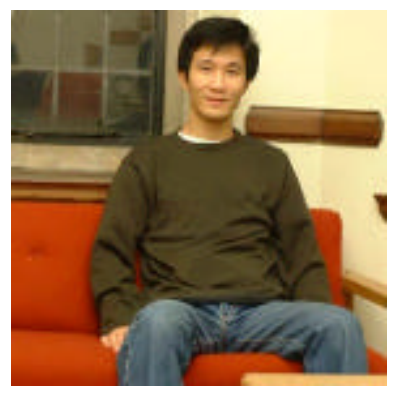

(a) Visual image

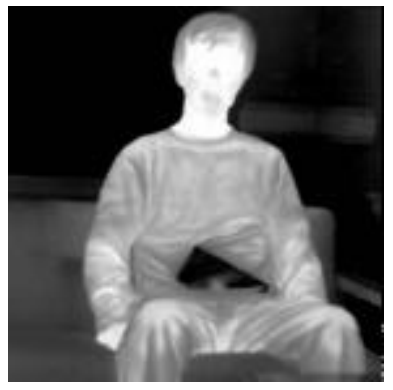

(b) IR image
Figure 1. CWD application

A variety of image fusion techniques have been developed. They can be roughly divided into two groups, multiscale-decomposition-based (MDB) fusion methods and non-multiscale-decomposition-based (NMDB) fusion methods. MDB image fusion schemes have been categorized by Zhang and Blum [4]. Typical MDB fusion methods include pyramid based methods, discrete wavelet transform based methods, and discrete wavelet frame transform based methods. Typical NMDB methods include adaptive weight averaging methods, neural network based methods, Markov random field based methods, and estimation theory based methods. Most of the mage fusion work has been limited to monochrome images. However, based on biological research results, the human visual system is very sensitive to colors. To utilize this ability, some researchers map three individual monochrome multispectral images to the respective channels of an RGB image to produce a false color fused image. In many cases, this technique is applied in combination with another image fusion procedure. Such a technique is sometimes called color composite fusion. Another technique is based on opponent-color processing which maps opponentsensors to human opponent colors (red vs. green, blue vs. 


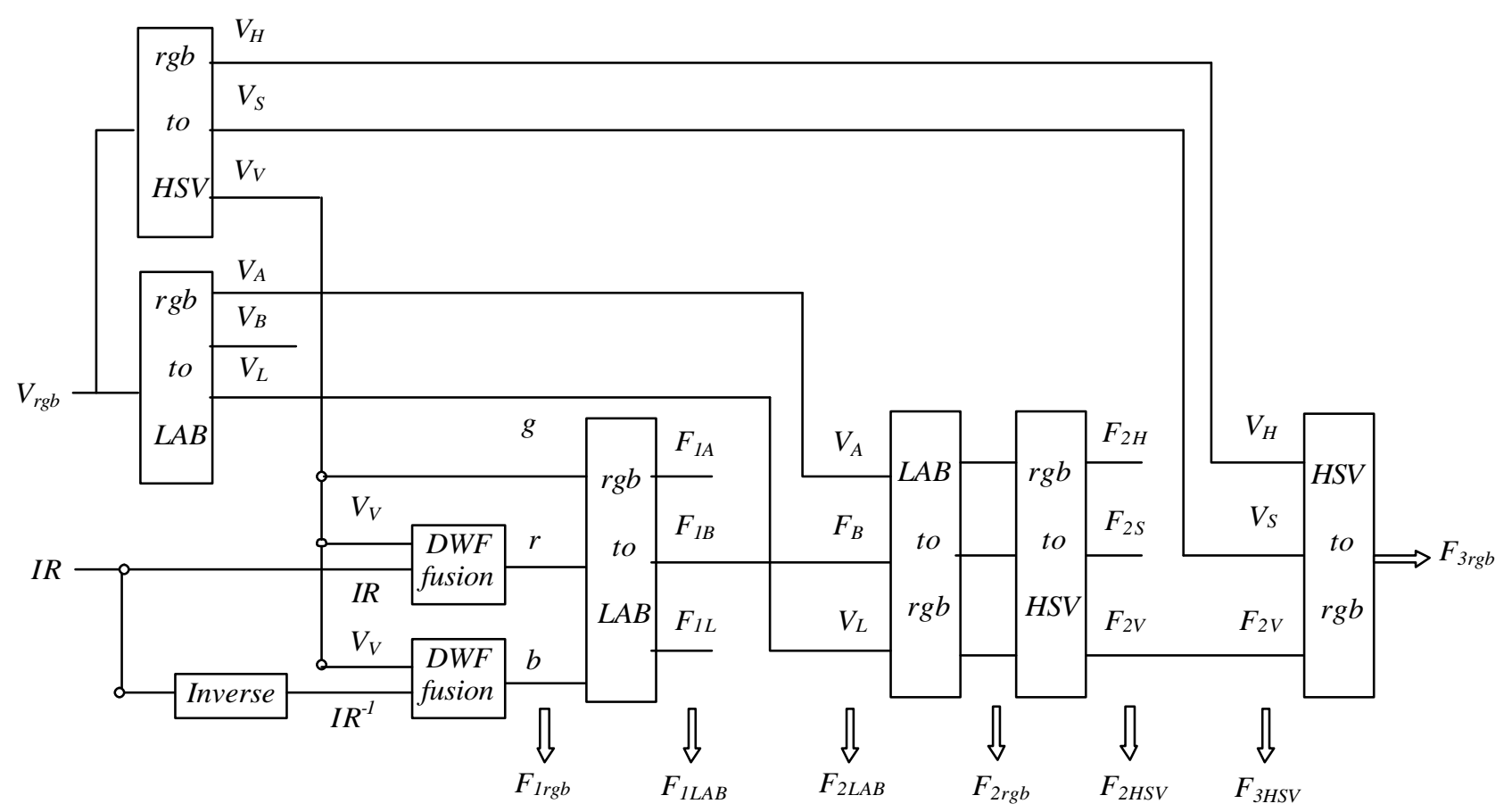

Figure 2. Color image fusion for CWD

yellow). For example, Waxman, Aguilar et. al [5-7] use a neural network to fuse a low-light visible image and a thermal IR image to generate a three-channel false color image used for night operation. In addition, Aguilar [8] has extended their work to fuse multi-modality volumetric medical imagery.

In this paper, we present a new technique to fuse a color visual image with a corresponding IR image for a CWD application. Using the proposed method the fused image will maintain the high resolution and the natural color of the visual image while incorporating any concealed weapons detected by the IR sensor.

The paper is organized as follows. Section 2 describes the proposed image fusion method for our CWD application. Section 3 presents the experimented results which demonstrate the feasibility of the proposed fusion technique and Section 4 summarizes the paper.

\section{Color Image Fusion Methodology}

The proposed color image fusion algorithm is illustrated in Figure 2. The fusion algorithm consists of several steps which will be explained in detail in the following.

Firstly, the input visual image denoted as $V_{r g b}$ is transformed from RGB color space (R: red, G: green, B: blue) into HSV (denoted as $V_{h s v}$ ) color space (H: hue, S: saturation, V: brightness value). HSV color space is used for the subsequent processing since it is well suited for describing colors in terms that are closely related to human interpretation. Further HSV allows a decoupling of the intensity component from the color-carrying information in a color image [9]. The $V$-channel of the visual image, which represents the intensity of the image, will be used in the fusion. The other two channels, the $H$-channel and the $S$ channel, carry color information. These two channels will be used to give the fused image a natural color which is similar to the color of the visual image. Besides the HSV color space, the LAB color space is also used. LAB color space is a uniform color space defined by the CIE (International Commission on Illumination). A color is defined in the LAB space by the brightness $L$, the redgreen chrominance $A$, and the yellow-blue chrominance $B$. This color space is also used for modifying the color of the fused image.

In the fusion step, both the original IR image and the reverse polarity of the IR image are used. The motivation for doing this is that, the concealed weapon is sometimes more evident in the IR image with reverse polarity. The $V$ channel of the visual image in HSV color space (denoted as $V_{V}$ ) is not only fused with the IR image (denoted as IR), but it is also fused with the reverse polarity IR image (denoted as $I R^{-1}$ ). The discrete wavelet frame (DWF) based fusion approach is used in both the fusion operations.

The DWF based method is one of many possible multiscale-decomposition-based (MDB) fusion methods. It consists of three main steps. First, each source image is decomposed into a multiscale representation using the DWF transform. Then a composite multiscale 
representation is constructed from the source representations and a fusion rule. Finally the fused image is obtained by taking an inverse DWF transform of the composite multiscale representation. The DWF is an overcomplete wavelet decomposition. Compared to the standard discrete wavelet transform (DWT), the DWF is a shift invariant signal representation which can be exploited to obtain a more robust image fusion method [4]. This property of DWF is important for fusing a visual and an IR image. An image fusion scheme which is shift dependent is undesirable in practice due to its sensitivity to misregistration. For the case of fusing a visual and an IR image, it is difficult to obtain perfect registration due to the different characteristics of visual and IR images. Hence, for our application, we use the DWF as the multiscale transform. The fusion rule we used is : choose the average value of the coefficients of the source images for the low frequency band and the maximum value of the coefficients of the source images for the high frequency bands. We use this typical fusion rule just to illustrate the feasibility of the proposed method of fusing a color image with an IR image. Other fusion rules can also be employed. At last, the fused image is produced by applying the inverse DWF.

After obtaining the two gray-level fused images, a color RGB image is obtained by assigning the $V$ channel of the visual image in HSV color space (denoted as $V_{V}$ ) to the green channel, the fused image of $V_{V}$ and $I R$ to the red channel, and the fused image of $V_{V}$ and $I R^{-1}$ to the blue channel. The resulting color image is called a pseudo color image $\left(F_{1 r g b}\right)$. The idea for the color mappings employed after the DWF fusion operations is motivated by the opponent color processing work by Aguilar and Waxman [5-7]. However, their specific approach is somewhat different. In fact, in their approach, shunting neural networks are employed.

The next step is to attempt to give the pseudo color image a more natural color (close to the color of the original visual image). Note that the cold regions (such as weapons) appear as bright regions in the reverse polarity of the IR image $\left(R^{-1}\right)$, and thus they appear as bright regions in the fused image $F_{l b}$ (obtained by fusing $V_{V}$ and $I R^{-1}$ ) also. Since the fused image $F_{l b}$ is assigned to the blue channel, the cold regions will be shown in blue color in the pseudo color image $F_{1 r g b}$, which is reasonable. In order to not only maintain the natural color of the visual image but also keep the complimentary information from the IR image (concealed weapon), the following procedure is applied to the pseudo color image $F_{l r g b}$. First, both the pseudo color image $F_{1 r g b}$ and the visual image $V_{r g b}$ are transformed from RGB color space into LAB color space (denoted as $F_{I L A B}$ $\left(F_{I L}, F_{I A}, F_{I B}\right)$ and $V_{L A B}\left(V_{L}, V_{A}, V_{B}\right)$, respectively). Then a new image $F_{2 L A B}\left(F_{2 L}, F_{2 A}, F_{2 B}\right)$ is obtained using the following method

$$
\left(F_{2 L}, F_{2 A}, F_{2 B}\right)=\left(V_{L}, V_{A}, F_{1 B}\right)
$$

That is, the $\mathrm{L}$ and $\mathrm{A}$ channels of $F_{I L A B}$ are replaced by the $\mathrm{L}$ and A channels of visual image $V_{L A B}$ respectively. Then the image $F_{2 L A B}$ is transformed from LAB color space into RGB color space to obtain the image $F_{2 r g b}$. In the LAB color space, the channel $L$ represents the brightness, the channel $A$ represents red-green chrominance, and the channel $B$ represents yellow-blue chrominance. Hence, by using the replacement given in above equation, the color of the image $F_{2 r g b}$ will be closer to the color of the visual image while incorporating the important information from the IR image (concealed weapon). However there is still some room to improve the color of the image $F_{2 r g b}$ to make it more like the visual image in the background and for the human body region. This is most easily achieved by utilizing the $\mathrm{H}$ and $\mathrm{S}$ components of the visual image $V_{H S V}$ in the HSV color space since the channels $\mathrm{H}$ and $\mathrm{S}$ contain color information $(\mathrm{H}$ : hue of the color, $\mathrm{S}$ : saturation of the color). Therefore, in the next step of color modification, first the image $F_{2 r g b}$ is converted into HSV color space $\left(F_{2 H S V}\left(F_{2 H}, F_{2 S}, F_{2 V}\right)\right)$, then a new image $F_{3 H S V}\left(F_{3 H}, F_{3 S}, F_{3 V}\right)$ is obtained by carrying out the following procedure,

$$
\left(F_{3 H}, F_{3 S}, F_{3 V}\right)=\left(V_{H}, V_{S}, F_{2 V}\right)
$$

That is, the $\mathrm{H}$ and $\mathrm{S}$ channel of $F_{2 H S V}$ are replaced by the $\mathrm{H}$ and $\mathrm{S}$ channel of $V_{H S V}$ respectively.

\section{Experimental Tests}

In order to illustrate the efficiency of the proposed color image fusion method for our CWD application, a dozen images were used in the experimental tests. Due to space limitations, only five of them and the corresponding fusion results are presented here which are shown in Figures 3-7, respectively. These test images were selected to test the fusion algorithm under various different conditions including different positions for the person, different colored clothing, different positions of the weapon, and different shapes of the weapon. For all the cases, the visual and IR images have been registered.

Figure 3(a) shows the original color visual image $V_{r g b}$. Figure 3(b) shows the gray-level visual image $V_{V}$, the $\mathrm{V}$ channel of the visual image $V_{H S V}$ in HSV color space, which is mapped in to the green channel of pseudo color image $F_{l r g b}$. Figure $3(\mathrm{c})$ shows the IR image. In the IR image, the concealed weapon is detected and shown in dark intensity due to its low temperature compared to the human body. It should be noted that, some areas of the background are also shown in low intensity (having similar gray color as the region of interest) because of their low thermal emissivity. Figure 3(d) shows the reverse polarity of the IR image. Figure 3(e) shows the fused image obtained from fusing the gray-level visual image $V_{V}$ and the IR image using a DWF multiscale representation $\left(F_{11}\right)$. In this image, we can identify the concealed weapon (coming from IR image) and the human's face. However the 
background is dark and not as clear as the gray-level visual image. This results from the low intensity of the background in the IR image and the use of averaging of low frequency bands. This fused image is being mapped into the red channel of the pseudo color image $F_{1 r g b}$. Figure 3(f) shows the fused image obtained from fusing the graylevel visual image $V_{V}$ and the reverse polarity IR image using a DWF multiscale representation $\left(F_{12}\right)$. Compared to Figure 3(e), the background is clearer but the human body is unclear due to its low intensity in the reverse polarity IR image. This fused image is being mapped into the blue channel of pseudo color image $F_{l r g}$. Figure $3(\mathrm{~g})$ shows the pseudo color fused image $F_{\text {lrgb }}$ (red channel: $F_{1 l}$, green channel: $V_{V}$, blue channel: $\left.F_{12}\right)$. Compared to the fused images shown in Figure 3(e) and 3(f), the background, the human face, and the concealed weapon are all clear in this pseudo color image. However, the color is quite different from that of the visual image. Figure 3(h) shows the color image after adjustment in LAB color space $\left(F_{2 r g b}\right)$. The color of this image is closer to the color of the visual image than $F_{\text {lrgb. }}$. As discussed before, cold regions, such as the concealed weapon and some areas of the background, will be shown in blue. Figure 3(i) shows the color image after additional adjustment in the HSV color space $F_{3 r g}$. The color of $F_{3 r g b}$ is very close to the color of visual image shown in Figure 3(a) and $F_{3 r g b}$ provides a good depiction of the concealed weapon. Figure 3(j) shows $F_{3 v}$, the V channel of the image $F_{3 H S V}$, which is obtained by converting the fused color image $F_{3 r g b}$. It is obvious that this image is better than either of the gray level fused images shown in Figure 3(e) and 3(f).

Examples for several other visual and IR image pairs are shown in Figure 4 through Figure 7. The proposed color fusion method works very well for images where the weapon is concealed under non-white color clothes as illustrated by Figures 4 and 5 . h Figure 6, we give an example of a human wearing a white shirt with the weapon concealed under it. By inspecting the fused image $F_{3 r g b}$ shown in Figure 6(g), we can still identify the location of the concealed weapon although the contrast is not as good as for the other cases. It is worth noting that, in the fused image, the cold regions inside the area of human body will have different color from the same place in the visual image. That's why the concealed weapon is shown in different color from the surrounding human body part. However, some parts of clothing near the weapon may not have contact with the human body and thus they may have a similar gray-level as the concealed weapon. One such example is shown in Figure 7. For this case, the color of trousers in the fused image is not as close to those in the visual image as for the other cases. However, we can still identify the location of the weapon. It should be pointed out that, one advantage of the proposed fusion method is that for the whole procedure, no parameter tuning or training is needed which means we don't need to

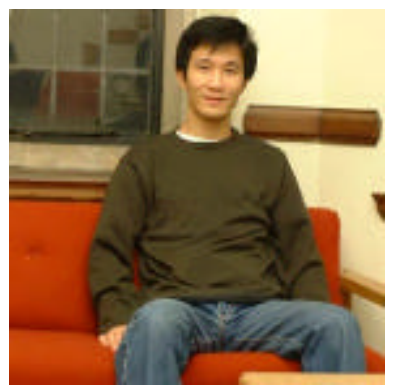

(a) Visual image

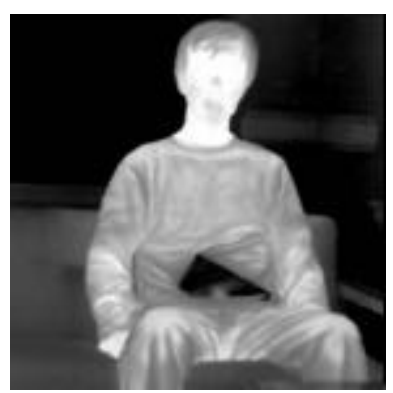

(c) IR image

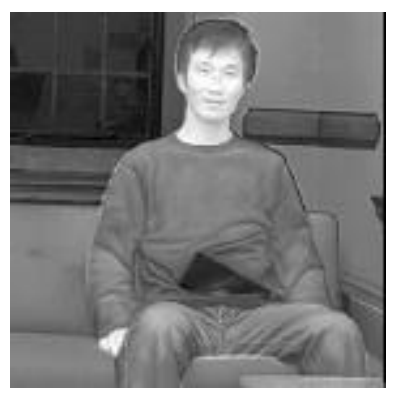

(e) Fused image $-r$ channel

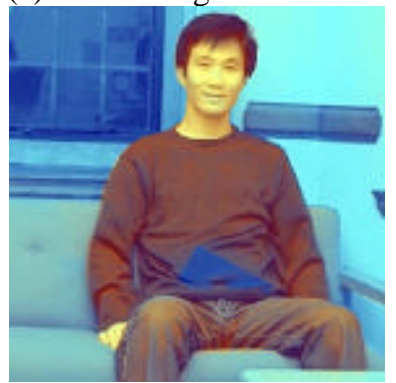

(g) Fused image $\left(F_{1 r g b}\right)$

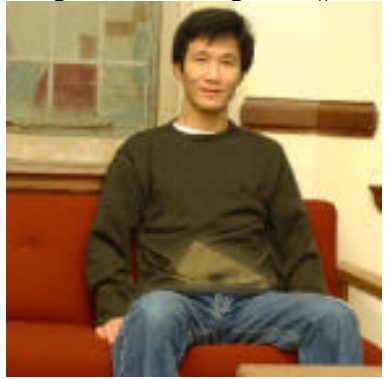

(i) Fused image $\left(F_{3 r g b}\right)$.

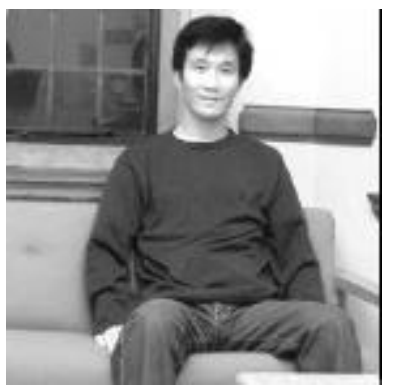

(b) Visual image - V/g

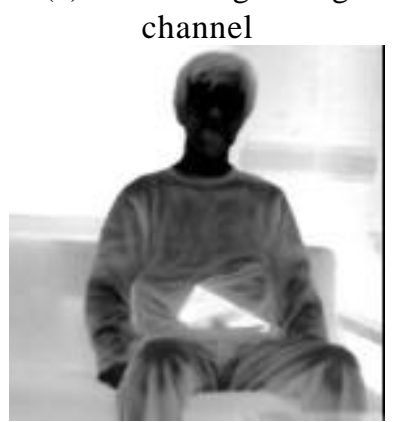

(d) IR image (Reverse polarity)

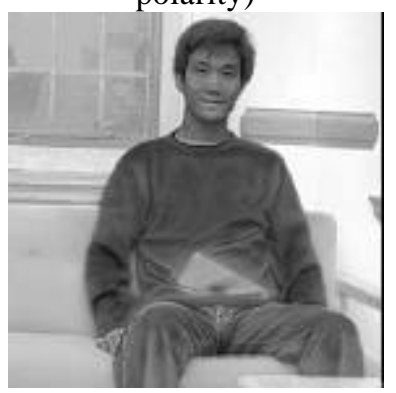

(f) Fused image $-b$ channel

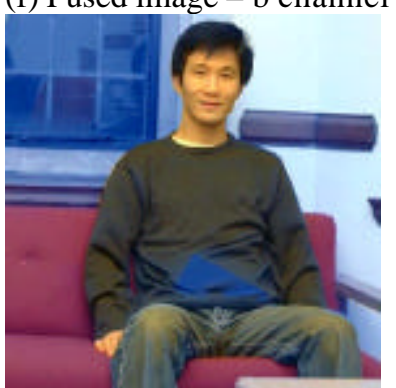

(h) Fused image $\left(F_{2 r g b}\right)$

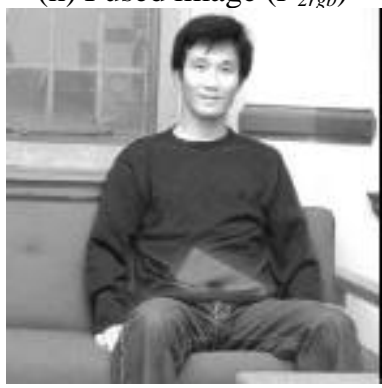

(j) Fused image $\left(F_{3 v}\right)$
Figure 3 Fusion Result - 1 
pre-use some images to find a good set of parameters that would perform well first.

We demonstrated the feasibility of the proposed fusion algorithm for detecting the concealed weapon under a human's clothes (shown in Figure 3 to 7). The proposed algorithm can also be applied for detecting a concealed weapon hidden under clothes or in bags without a human present. One example and the corresponding fused image are shown in Figure 8. It can be seen that the fused images are also useful for these cases.

\section{Conclusion}

In this paper, we proposed a new color image fusion method for a concealed weapon detection application where we fuse visual and IR images to provide a fused image that provides a detailed description of the people in the scene and any hidden weapons detected by the IR image. The utility of the proposed method is demonstrated in the experiment tests.

\section{References}

[1] P. K. Varshney, H. Chen, L. C. Ramac, M. Uner, Registration and fusion of infrared and millimeter wave images for concealed weapon detection, in Proc. of International Conference on Image Processing, Japan, vol. 3, Oct. 1999, pp. 532-536.

[2] M. K. Uner, L. C. Ramac, P. K. Varshney, Concealed weapon detection: An image fusion approach, Proceedings of SPIE, vol. 2942, pp. 123-132, 1997.
[3] Z. Zhang, R. S. Blum, A region-based image fusion scheme for concealed weapon detection, Proceedings of 31st Annual Conference on Information Sciences and Systems, pp. 168-173, Baltimore, MD, Mar 1997.

[4] Z. Zhang, R. S. Blum, A categorization of multiscaledecomposition-based image fusion schemes with a performance study for a digital camera application, Proceedings of IEEE, vol. 87, no. 8, pp. 1315-1326, 1999.

[5] A. M. Waxman, M. Aguilar, R. A. Baxter, D.A. Fay, D. B. Ireland, J. P. Racamato, W. D. Ross, Opponent-color fusion of multi-sensor imagery: visible, IR and SAR, Proceedings of IRIS Passive Sensors, vol.1, pp. 43-61, 1998.

[6] M. Aguilar, D. A. Fay, W. D. Ross, A. M. Waxman, D. B. Ireland, J. P. Racamato, Real-time fusion of low-light CCD and uncooled IR imagery for color night vision, Proceedings of the SPIE, vol.3364, pp. 124-35, 1998.

[7] A. M. Waxman, M. Aguilar, D. A. Fray, D. B. Ireland, J. P. Racamato, Jr., W. D. Ross, J. E. Carrick, A. N. Gove, M. C. Seibert, E. D. Savoye, R. K. Reich, B. E. Burke, W. H. McGonagle, D. M. Craig, Solid-state color night vision: fusion of low-light visible and thermal infrared imagery, Lincoln Laboratory Journal, vol. 11, no. 1, pp. 41-60, 1998.

[8] M. Aguilar, and J. R. New, Fusion of multi-modality volumetric medical imagery, ISIF 2002, pp. 1206-1212.

[9] R. C. Gonzalez, R. E. Woods, Digital Image Processing, Second Edition, Prentice Hall, New Jersey 2002.

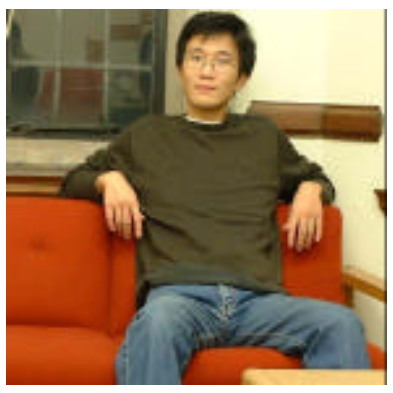

Figure 4: (a) Visual image

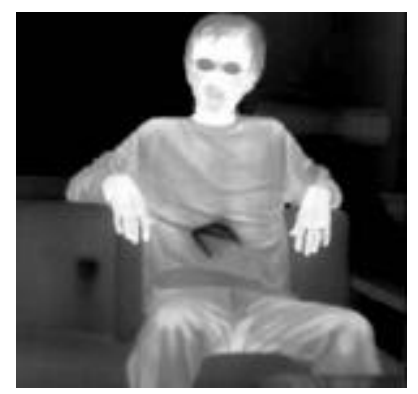

(b) IR image

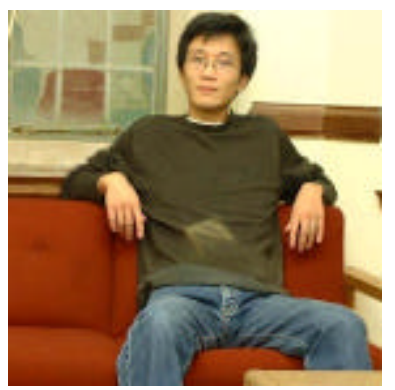

(c) Fused image 


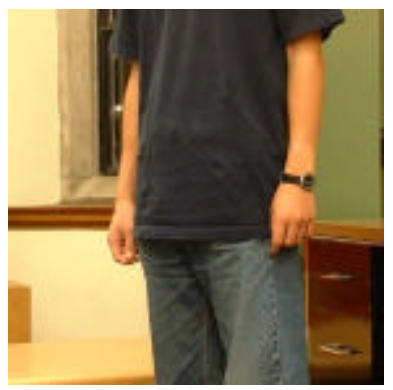

Figure 5: (a) Visual image

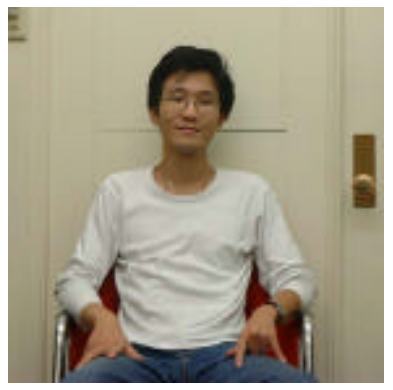

Figure 6: (a) Visual image

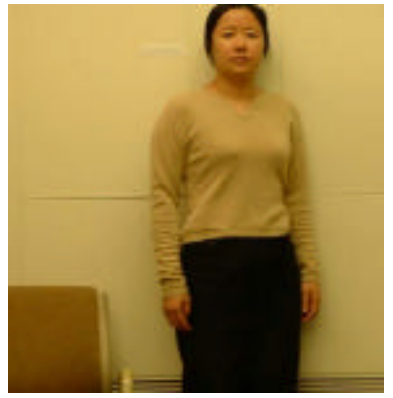

Figure 7: (a) Visual image

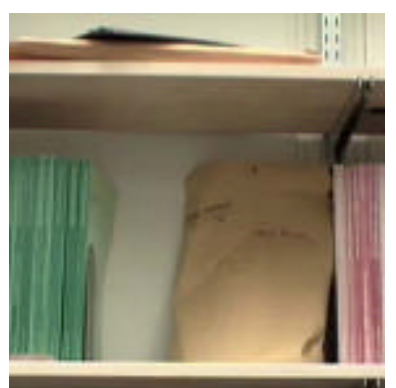

Figure 8: (a) Visual image

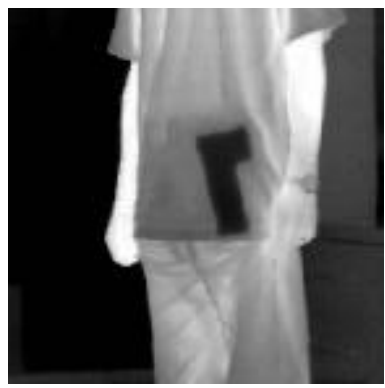

(b) IR image

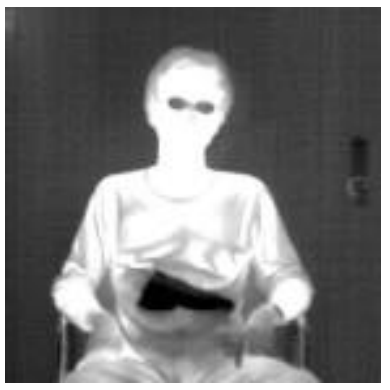

(b) IR image

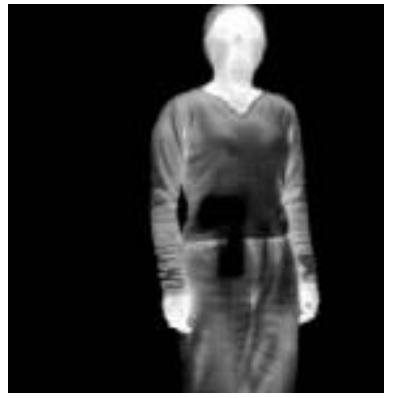

(b) IR image

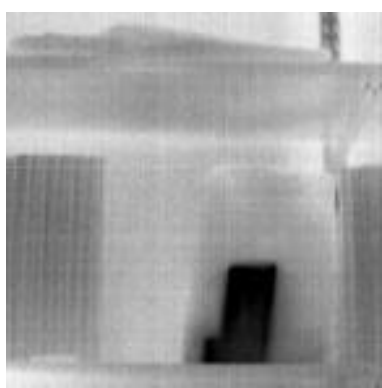

(b) IR image

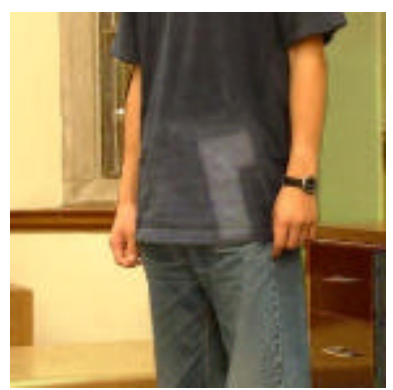

(c) Fused image

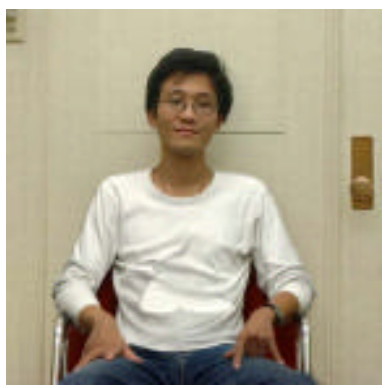

(c) Fused image

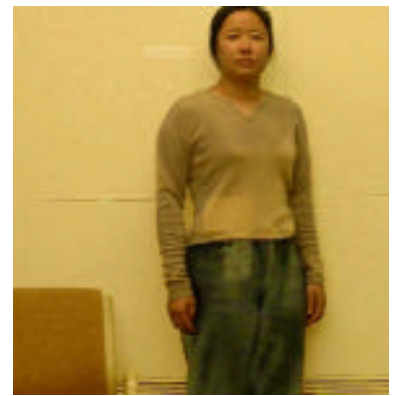

(c) Fused image

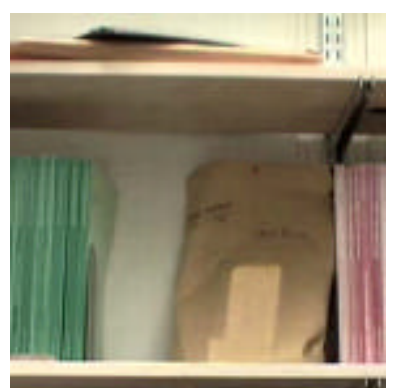

(c) Fused image 